\title{
USO DE PSICOFÁRMACOS E A IMPORTÂNCIA DO FARMACÊUTICO NA ESTRATÉGIA SAÚDE DA FAMÍLIA ${ }^{1}$

\author{
USE OF PSYCHOTROPIC DRUGS AND THE IMPORTANCE OF \\ PHARMACIST IN FAMILY HEALTH STRATEGY
}

\author{
Andressa Caroline Loebens Diel ${ }^{2}$ e Karla Renata de Oliveira ${ }^{3}$
}

\section{RESUMO}

A Estratégia Saúde da Família contempla uma equipe multiprofissional, na qual pode fazer parte o farmacêutico. Os psicofármacos necessitam atenção especial quanto ao seu uso, devido reações adversas, risco de tolerância e dependência. $\mathrm{O}$ objetivo deste estudo foi compreender o conhecimento dos usuários sobre os psicofármacos utilizados, a adesão desses sujeitos à terapia e o tempo de uso dos medicamentos, além de demonstrar a importância do farmacêutico em uma ESF de um município do interior do Rio Grande do Sul. Trata-se de um estudo descritivo, exploratório, com abordagem qualitativa. Os medicamentos mais utilizados foram amitriptilina, fluoxetina e diazepam, sendo que a maioria dos usuários não sabiam identificar seus medicamentos no que se refere à indicação terapêutica. Muitos demonstraram necessitar de auxílio em seus tratamentos, não tendo autonomia na administração dos medicamentos; quanto à adesão verificou-se falhas relacionadas principalmente ao esquecimento e o uso se deve principalmente ao efeito calmante, de relaxamento e indução do sono. Muitos utilizam os medicamentos há vários anos, o que repercute de forma negativa na sua saúde e indica um baixo conhecimento desses sujeitos com relação ao tratamento medicamentoso. Dessa forma, o farmacêutico é importante na conduta da terapia medicamentosa, qualificando os serviços da Estratégia Saúde da Família.

Palavras-chave: Atenção farmacêutica, Conhecimento, Uso racional de medicamentos.

\section{ABSTRACT}

Family Health Strategy contemplates a multiprofessional team, in which the pharmacist can be part. Psychotropic drugs need special attention for their use due to adverse reactions, dependence and tolerance. The aim of this study was to understand the knowledge of users about psychotropic drugs, the adherence to therapy, and the usage time of drugs, in addition to demonstrating the importance of the pharmacist. This is a descriptive, exploratory study with a qualitative approach. The most commonly used drugs were amitriptyline, fluoxetine and diazepam, once most users did not know how to identify their medications with respect to the therapeutic indication. Many people have shown the need for help in their treatments, and do not have autonomy in the administration of the drugs; the adherence failed mainly due to problems of forgetting and the use of the drugs was mainly due to soothing sensation, relaxation and induction of sleep. Many people have been using the drugs for several years and can be dependent, indicating low knowledge of these subjects regarding drug treatment. In this way, the pharmacist is important in the conduct of the drug therapy, qualifying the services of Family Health Strategy.

Keywords: Knowledge, Pharmaceutical attention, Rational use of drugs.

\footnotetext{
${ }^{1}$ Trabalho de Conclusão de Residência Multiprofissional em Saúde da Família

${ }^{2}$ Graduada em Farmácia pela Universidade Federal de Santa Maria. Especialista em Saúde da Família pela Universidade Regional do Noroeste do Rio Grande do Sul. E-mail: andressa.diel@hotmail.com

${ }^{3}$ Orientadora. Graduada em Farmácia pela Universidade Federal de Santa Maria. Mestre em Ciências Biológicas/ Bioquímica pela Universidade Federal do Rio Grande do Sul. E-mail: karla.deoliveira71@gmail.com
} 


\section{INTRODUÇÃO}

A Atenção Primária à Saúde (APS) é a principal porta de entrada ao Sistema Único de Saúde (SUS) e oferta serviços para a promoção, prevenção e recuperação da saúde (BRASIL, 2017). Nesse sentido, a Estratégia Saúde da Família (ESF), visa desenvolver a APS de maneira multiprofissional, com foco no atendimento às famílias e comunidade, e não só ao usuário em seu contexto singular (MIASSO; FILHO; BORGES, 2017).

Em um município do interior do Rio Grande do Sul, a APS é desenvolvida por meio da [omitido para submissão] que possui autonomia administrativa e financeira, equiparando-se a secretaria de saúde municipal ([omitido para submissão], 1995). Em parceria com a Universidade [omitido para submissão] desenvolve o Programa de Residência Multiprofissional em Saúde da Família (PRMSF), visando à formação de profissionais aptos para atuarem na APS. Considerando o caráter multiprofissional, são ofertadas vagas para diversas profissões, dentre elas, uma para farmacêutico, importante norteador de ações em saúde relacionadas aos medicamentos e ao tratamento farmacológico dos usuários do serviço municipal de saúde.

$\mathrm{Na}$ área farmacêutica, as inovações referentes à ciência e tecnologia, contribuem para a ampliação de oferta de medicamentos no mercado, auxiliam nas condições agudas e crônicas de saúde e elevam a expectativa de vida da população (GOMES et at., 2014). Porém, o número elevado de prescrições e o possível abuso de fármacos, em especial os psicofármacos, geram preocupação devido seus impactos sociais, econômicos e suas implicações nem sempre positivas na saúde (GRUBER; MAZON, 2014; GUERRA et al., 2013).

O baixo conhecimento sobre o tratamento farmacológico é um dos fatores que pode comprometer a segurança e efetividade do tratamento com psicofármacos (NICOLINO et al., 2011). Estudos destacam a preservação do conhecimento do paciente referente à sua doença como um fator protetor para a manutenção do tratamento farmacológico (CARDOSO et al., 2011) e quando este é realizado por autoadministração, o conhecimento sobre o esquema terapêutico é fundamental para dar seguimento ao tratamento (SOUZA et al., 2013).

Já a adesão ao tratamento medicamentoso é um processo que está relacionado com as características do usuário, costumes e percepção sobre a doença, além de aspectos do próprio tratamento, como acesso, tempo de espera e relacionamento do usuário com a equipe da ESF. Além disso, a falta de adesão é um problema de cuidado em saúde que limita a eficácia do tratamento, tornando maiores as taxas de recidivas da doença, internações hospitalares e mortes precoces (GOWDA, 2017).

Quando utilizados de forma irracional, os medicamentos podem provocar reações adversas, em especial, os psicofármacos, pois geram alterações no organismo, como mudanças de comportamento, emoções, pensamentos e percepções (GUERRA et al., 2013) e em alguns casos, causam tolerância e dependência, o que pode gerar danos à saúde do usuário, além de gastos desnecessários 
em saúde pública. Apesar do uso nem sempre racional, também deve-se considerar que existem demandas para a utilização destes, devido transtornos mentais, o que requer alguns cuidados tanto por parte do usuário, quanto da equipe de saúde que o acompanha (SANTO et al., 2009).

Neste contexto, este estudo teve como objetivo compreender o conhecimento dos usuários sobre os psicofármacos utilizados, a adesão desses sujeitos à terapia e o tempo de uso dos medicamentos, além de demonstrar a importância do farmacêutico em uma ESF de um município do interior do Rio Grande do Sul.

\section{METODOLOGIA}

Trata-se de um estudo descritivo, exploratório, com abordagem qualitativa, realizado em janeiro de 2018, em uma ESF de um município no interior do Rio Grande do Sul.

O município possui população estimada em 72.753 pessoas (IBGE, 2017) e destaca-se pela alta qualidade nos serviços da APS, sendo referência em saúde pública para o Estado. O serviço municipal de saúde está organizado em 17 ESF, dessas, três possuem unidades avançadas de saúde no interior do município (PREFEITURA MUNICIPAL DE SANTA ROSA, 2017). A ESF selecionada para o estudo foi local de trabalho durante dois anos, período em que a pesquisadora realizou especialização em Saúde da Família, na modalidade Residência.

Os participantes da pesquisa foram selecionados a partir do registro da dispensação de psicofármacos referente ao mês de dezembro de 2017. Todos os usuários que retiraram esses medicamentos no serviço público municipal no período do estudo foram incluídos em um sorteio aleatório e os sorteados procurados em seus domicílios e convidados a responder um questionário semiestruturado. Além do sorteio, foram utilizados critérios de inclusão na pesquisa: ter idade maior ou igual a 18 anos, ser morador da área urbana adstrita a ESF e não apresentar dificuldades cognitivas para responder as questões ou problemas na fala. Os usuários selecionados que aceitaram participar da pesquisa, assinaram o Termo de Consentimento Livre e Esclarecido (TCLE) e os questionários foram aplicados até a saturação das falas dos entrevistados.

Com relação ao instrumento de coleta de dados, a primeira parte incluía dados sociodemográficos (sexo, idade, escolaridade, estado civil e profissão) e na segunda, os usuários foram questionados em relação ao seu conhecimento sobre os medicamentos em uso, quando foi solicitada a apresentação da embalagem primária de cada medicamento para a identificação dos mesmos; além de questionados sobre a necessidade de uso, tempo de tratamento e autonomia na utilização. Sobre a adesão a terapia medicamentosa, os usuários responderam a uma questão específica, método citado por Coutinho; Souza (2011) que consiste em atribuição de nota de um a cinco (onde um é o menos aderente e cinco o mais aderente).

As entrevistas foram gravadas com auxílio de um aparelho celular, com o aplicativo "Gravador de Voz Fácil” e posteriormente, transcritas para melhor compreensão e análise. Os dados foram 
organizados com base no método de análise de conteúdo de Minayo. Foi realizada leitura e exploração do material obtido através do questionário, categorização das respostas e interpretação dos dados (MINAYO, 2010). A definição das categorias levou em consideração a semelhança das respostas dos sujeitos e os objetivos do projeto. A pesquisa envolveu 18 usuários, identificados no decorrer do texto por números de 1 a 18 .

O projeto de pesquisa foi aprovado pelo Núcleo de Ensino e Pesquisa (NEP) da Fundação Municipal de Saúde de Santa Rosa, parecer n ${ }^{\circ}$ 2739/2017 e pelo Comitê de Ética em Pesquisa (CEP) da Universidade Regional do Noroeste do Estado do Rio Grande do Sul, parecer consubstanciado $\mathrm{n}^{\mathrm{o}} 2.456 .609 / 2017$.

\section{RESULTADOS E DISCUSSÃO}

Dos 18 entrevistados, a maioria são mulheres, com idades entre 22 e 77 anos, baixo grau de escolaridade (fundamental incompleto), baixo vínculo empregatício (aposentados, do lar ou com auxílio saúde) e predomínio do estado civil casado.

Dos participantes, a maioria utilizava amitriptilina $25 \mathrm{mg}$, fluoxetina $20 \mathrm{mg}$ e diazepam 10 $\mathrm{mg}$, sendo que, alguns utilizavam os três medicamentos concomitantemente, ou ainda, utilizavam outros medicamentos para tratar condições crônicas de saúde. Diante disso, é possível inferir que a maioria dos entrevistados encontra-se polimedicado.

A análise das falas deu origem a três categorias temáticas, que expressam o significado do uso dos psicofármacos a partir da visão dos entrevistados, e permitem identificar os fatores que interferem no uso adequado e na adesão ao tratamento com esses medicamentos.

\section{Conhecimento dos usuários em relação aos medicamentos utilizados}

Nesta categoria, os entrevistados foram questionados sobre os medicamentos que utilizavam e sua finalidade.

Eu acho que é pro coração, não é (fluoxetina)? Não sei se é. Não sinto nada diferente quando tomo ele, não observo sabe, não sinto nada. De repente às vezes dá uma coisa, mas a gente não sente, porque eu sempre tô assim meio ruim [...] (Entrevistado 5).

Eu não sei pra que serve, eu sei que me sinto bem, sou muito braba e me sinto bem com ele [...] (Entrevistado 4)

A maioria dos entrevistados demonstrou desconhecimento no que se refere à indicação terapêutica do medicamento em uso, alguns relacionavam a tristeza, agitação e ansiedade. Evidenciou-se 
também, que o número de medicamentos em uso é um fator que dificulta na identificação da finalidade terapêutica. Aqueles que identificaram a ação dos medicamentos, estabeleceram relação entre uma das embalagens do produto (primária ou secundária) e sua doença.

Além do baixo conhecimento em relação a seu tratamento, alguns usuários manifestaram pouco interesse em conhecer sobre seus medicamentos devido à crença do conhecimento médico, “ah, eu tomo porque o médico mandou” (entrevistados 1, 3, 5, 8), o que diminui o comprometimento dos usuários com seu tratamento:

Eu acho que pra mim faz bem, né? Eu tenho vários problemas né, há muito tempo que eu tomo esses aí, então eu acho que pra mim faz bem, por isso tem que tomar. Se o doutor deu pra mim tomar é porque algum bem ele faz né? Não sei dizer bem o que é... não sei pra quê servem esses remédios... Entrevistado 7.

As falas vão ao encontro de outros estudos. Em pesquisa desenvolvida em Unidade de Saúde de Teresina, Piauí, investigou-se o conhecimento de idosas sobre o uso de psicofármacos, algumas identificaram a indicação dos medicamentos, enquanto outras não sabiam informações e não havia interesse sobre os fármacos, pois acreditavam que o saber médico era inquestionável e não lhes fazia sentido buscar explicações sobre os medicamentos (PRUDÊNCIO; NOGUEIRA, 2013).

Dessa forma, é importante destacar a corresponsabilização do sujeito em seu tratamento. Além do diagnóstico e da prescrição pelo profissional de saúde, é necessário que o usuário assuma responsabilidade na continuidade da sua terapia. No que se refere à atenção à saúde, o usuário é visto como participante ativo deste processo, com compromisso em seguir as orientações da prescrição (OENNING; OLIVEIRA; BLATT, 2011). Essa falta de conhecimento sobre os medicamentos pode resultar em falhas terapêuticas e abandono da terapia medicamentosa (CARVALHO et al., 2012) e por este motivo, esta responsabilidade pode auxiliar na redução dos efeitos negativos da doença, além de projetar o usuário ao enfrentamento de seus problemas e empoderamento de sua saúde (FIRMINO; JORGE, 2015).

Ainda, em vista do enfrentamento de problemas relacionados ao tratamento é importante que haja a percepção da doença pelo usuário, sendo necessário que ele identifique seu problema de saúde e o tratamento farmacológico correspondente (COUTINHO; SOUZA, 2011).

Contudo, as entrevistas revelam que quando o usuário desconhece a indicação terapêutica do medicamento, ele não se compromete em fazer o uso correto, já que desconhece os benefícios da terapia. O conhecimento, portanto, é um fator que auxilia na manutenção do tratamento e nesse sentido, o farmacêutico e a equipe de saúde exercem fundamental importância. A partir do acompanhamento individual, é possível promover uma reflexão sobre os benefícios do tratamento medicamentoso, comprometendo o usuário com relação à administração/adesão ao tratamento, o que é trabalhado a cada encontro posterior às orientações iniciais do profissional. 


\section{Adesão ao tratamento farmacológico e fatores associados}

Identificou-se relatos referentes às reações adversas decorrentes do uso dos medicamentos que interferem na adesão ao tratamento.

A amitriptilina amortece tudo, deixa a gente com a língua meio pesada, no outro dia é ruim pra conversar, né? (Entrevistado 3).

Ele (amitriptilina) vai amortecendo a boca, sabe? As vezes ataca o estômago bastante, mas fazer o que né, tem que tomar, sou obrigada a tomar. A fluoxetina me dava muita dor de cabeça, aí tive que parar (Entrevistado 6).

O entrevistado 3 relata uma reação adversa e a forma com que essa interfere na convivência diária com outras pessoas. Já o entrevistado 6 percebeu reações com uso de dois medicamentos diferentes, e suspendeu o uso daquele que gerava maior desconforto. Dessa forma as reações adversas podem contribuir para a redução da adesão ao tratamento farmacológico, em geral pelo desconforto que gera nos usuários.

A amitriptilina eu tomo só de noite, e um só... tô tomando só um, porque dá muita bobeira na gente...tomo antes de dormir, dá sonolência, a boca as vezes amortece, da tipo uma ansiedade as vezes [...] (Entrevistado 11).

As vezes tenho azia tomando esses (fluoxetina e amitriptilina). Se eu tô mais em jejum, aí eu tenho que tomar um daqueles pro estomago, né? (omeprazol). (Entrevistado 12).

Percebe-se que a manifestação das reações adversas interfere no tratamento, em especial na posologia, modificada pelo próprio usuário. $\mathrm{O}$ entrevistado 11 que deveria utilizar três comprimidos de amitriptilina utiliza somente um pelo efeito prolongado da sonolência no dia posterior ao uso, e no caso do entrevistado 12 leva a prática da automedicação. Muitos, portanto, relataram alterar a posologia em decorrência dos efeitos indesejados. Esta pesquisa corrobora com o encontrado por Czarnobay (2015), que relacionou reações adversas como interferências no sono, agitação e inquietação ao uso de psicofármacos, e essas como fatores para a não adesão ao uso dessa classe de medicamentos.

Para Ferreira e colaboradores (2013), as reações adversas podem ser reduzidas ou evitadas se forem acompanhadas pelo farmacêutico, sendo que o profissional é capaz de avaliar o caso, proporcionar educação em saúde, avaliar interações, e interagir com o prescritor se necessário. $\mathrm{O}$ acompanhamento clínico do farmacêutico auxilia o usuário no esclarecimento de dúvidas, orientando-o quanto aos horários de administração de doses para a redução dos efeitos indesejados.

Quanto à autonomia relacionada ao uso dos medicamentos, a maioria dos usuários relatou utilizá-los de forma independente, porém, em grande parte das falas foram identificados esquecimento de doses: 
Não, não tomo sozinha porque às vezes eu me esqueço. Meu esposo ajuda, todo dia não, quando ele vê que tem dias que eu não me lembro muita coisa, dai ele diz "hoje vou ter que te ajudar", dai ele levanta e deixa (os medicamentos) e fala esse é assim, esse é assim [...] (Entrevistado 3).

Meu esposo ajuda, nunca tomo sozinha; ele e meu filho, né? Meu filho me dá de meio dia, e daí de manhã e de noite é meu marido, antes dele ir pro trabalho ele me medica. (Entrevistado 7).

As falas indicam a necessidade de auxílio dos familiares para a utilização dos medicamentos e a principal causa da falta de autonomia foi o esquecimento. Esta pode estar relacionada ao fato dos usuários serem polimedicados, o que os leva a confundir os medicamentos; o fato de haver múltiplas tomadas do mesmo medicamento durante o dia, além de que, quando se trata de idosos, há diminuição da memória.

A autonomia sobre a administração dos medicamentos é considerada condição de saúde e cidadania para o sujeito (SOARES; CAMARGO, 2007), dessa forma, é importante que o usuário identifique seus medicamentos, posologias, e horários de administração para a manutenção e empoderamento com relação à sua saúde. O conhecimento sobre o esquema terapêutico, portanto, é fundamental para dar seguimento ao tratamento (SOUZA et al., 2013).

Sobre o nível de adesão ao tratamento farmacológico, a maioria considerou-se aderente (nota 5) ou relataram que utilizavam quase todos os dias o medicamento (nota 4):

Acho que é o número 5...eu sempre tomo...é toda noite, eu gosto de tomar. Quando tá em falta eu não tomo, mas dai também não durmo. Ontem não dormi né, ainda passei ruim, tinha dor no estômago. (Entrevistado 2).

Pra amitriptilina eu acho que é o número 4, quase sempre. Dois dias não tomo, tomo três dias. Porque daí eu tenho que conversar um pouco, né? Isso aí não deixa nem a gente falar quase [...] (Entrevistado 3).

Cabe destacar que na autoavaliação, os entrevistados não consideraram falha ou falta de adesão o esquecimento de doses ou as alterações posológicas que relataram, o que superestima o resultado do teste utilizado.

Já nas situações em que o usuário demonstra interesse e adesão ao tratamento, percebe-se um certo grau de dependência desenvolvida em relação ao medicamento, em função do uso prolongado, identificado a partir de sintomas de síndrome de abstinência relatados pela utilização do fármaco, a fim de tratar sintomas físicos:

O número 5. Os dois (medicamentos) eu utilizo sempre... não tem como parar. Eu deixo do lado da cama parado pra eu lembrar. Se eu esqueço eu não durmo, não durmo mesmo, eu tenho que tomar pra eu poder dormir. (Entrevistado 6).

Se eu não tomar da uma reação... me dá uma falta... tremo, tremo, começo pensar besteira, já vejo logo que não dá... não vou tentar ficar sem... aí tem gente que diz que não toma todo dia, mas eu quero ficar bem. (Entrevistado 18). 
A necessidade de estar bem o tempo todo é um dos motivos para os usuários lembrarem de utilizar o medicamento. Os resultados estão de acordo com a pesquisa de Leite (2013), que quando há falta de adesão, percebe-se que esta não é intencional, e está relacionada com o esquecimento do medicamento (LEITE, 2013). A não adesão ao tratamento relaciona-se com diversos fatores, como idade, tipo da doença, efeitos adversos, horários de utilização, inclusive fatores relacionados ao profissional de saúde que acompanha o usuário.

Ainda, a falta do apoio da família foi descrita por Czarnobay (2015) como um dos principais fatores para a não adesão, tendo agravados os casos de usuários com limitações visuais e de leitura. Nesse sentido, a participação dos familiares torna-se essencial para a adesão do usuário, auxiliando-o na administração de seus medicamentos em seus horários e formas corretas (LEITE, 2013).

As reações adversas, autonomia e adesão aqui descritas também estão relacionadas com o conhecimento dos usuários sobre seu tratamento. Quando o usuário não sabe o que está utilizando, pode associar equivocadamente as reações manifestadas a algum medicamento ou pode abandoar o tratamento, por entender desnecessário seu uso. Se o benefício encontrado no medicamento for maior que as reações adversas, o usuário conscientiza-se da importância de utilizá-lo e passa a ter melhor adesão.

Compreender o objetivo do tratamento, a conduta terapêutica e os efeitos decorrentes configura-se, portanto, em um passo para o planejamento do autocuidado ação que pode ser desenvolvida pelo usuário em conjunto com o farmacêutico e a equipe multiprofissional (OLIVEIRA; LOPES; CASTRO, 2015).

\section{Tempo de tratamento farmacológico e riscos relacionados}

O tempo de tratamento com os medicamentos variou, mas a maioria relatou longo período de uso. Entre os usuários de amitriptilina, o tempo de uso variou entre um e 15 anos, enquanto para fluoxetina entre um mês e 10 anos. Quanto aos BDZ's, e o mais preocupante, foi à utilização de diazepam - os entrevistados relataram utilizar há 20, 24, e 30 anos e clonazepam - seis anos de uso.

Segundo Souto e colaboradores (2017), a insônia é uma das queixas mais frequentes na APS, sendo seus efeitos refletidos na sensação de prazer e bem-estar, o que faz com que os BDZ's sejam amplamente prescritos. Ainda de acordo com pesquisa de Firmino e colaboradores (2012), o uso de BDZ'S acima de seis meses é fator de risco para desenvolver tolerância e dependência. Para Farias e colaboradores (2015) o uso em idade avançada não é recomendado mais que quatro meses de tratamento, sendo que não há efeito terapêutico de alívio da ansiedade nesta idade. Verificou-se que a maioria dos entrevistados não consegue interromper o uso destes medicamentos, aparentemente, devido a efeitos de abstinência e dependência, e acreditam ter de utilizá-los para o resto da vida:

É necessário tomar porque eu não durmo sem a medicação. Tentei ficar sem, eu parei cinco dias... daí eu fui na doutora, ela disse que "não adianta, teu organismo tá viciado e vai tomar 
isso pro resto da vida"[...] Sem a medicação não dormia, caminhava dentro de casa... mas eu quero consultar mesmo, pra ver se não tem um diazepam mais forte [..]. (Entrevistado 14).

Se eu não tomo não durmo nada e não tenho sossego, tem que levantar, deitar, sentar. Eu deito e daí tomo meu diazepam, daí faz um sono até as 3 horas eu durmo, aí depois eu me acordo e não durmo mais, né? Mas eu dormindo até lá tá bom, né? (Entrevistado 2).

Os relatos dos usuários sobre a necessidade de uso dos BDZ's, refere-se ao "poder dormir", evidenciado em múltiplas entrevistas e mesmo fazendo uso do medicamento, muitos tem a indução inicial do sono, mas não conseguem manter a qualidade dele, nem dormem a noite toda. Necessitar de um "diazepam mais forte” pode indicar que o mesmo não está mais fazendo seu efeito terapêutico, associado ao uso há mais de 20 anos.

Para Alvarenga e colaboradores (2015), o início de uso do BDZ acontece pela prescrição médica, mas a continuidade do tratamento se dá por meio do resultado positivo ou negativo da experiência de sua utilização e como a maioria descreve sintomas iniciais positivos, seguem o tratamento. Além disso, o medo do retorno e intensificação dos sintomas manifestados anteriormente, determina a continuidade pelo uso do BDZ como observado a seguir:

Tentei ficar uns dois, três meses sem a medicação, mas aí tive que voltar, tive ansiedade bastante, não dormia, ficava muito agitado... tinha parado por conta, mas dai voltei no médico e ele receitou de novo, mas agora eu tô bem, tô tranquilo. Parar com esse remédio aí é bem difícil parar [...] (Entrevistado 15).

Quando há tentativa de suspender o uso do medicamento, reações físicas se manifestam, como a ansiedade e agitação descrita pelo entrevistado 15 , e mesmo que o medicamento não promova o efeito desejado, o usuário sente necessidade de continuar o uso para não sentir o efeito negativo da retirada.

A utilização de BDZ's a longo prazo gera preocupação em especial na população idosa. Dos cinco usuários que utilizam BDZ's, dois são idosos, com 65 anos e 73 anos. O risco encontra-se no fato dos idosos serem polimedicados, apresentarem capacidade cognitiva e de memória reduzidas, além do metabolismo mais lento, o que contribui para o maior risco de queda, em função da grande sedação proporcionada pelo medicamento (SILVA et al., 2015).

Segundo Solis (2016), a reestruturação das receitas, a garantia da consulta médica e o acompanhamento do tratamento são fatores que podem auxiliar na redução do uso irracional de BDZ's, assim como práticas alternativas como a higiene do sono, recursos de lazer e melhor alimentação tornam-se efetivos na qualidade de vida dos usuários.

Segundo Oliveira e colaboradores, o papel do farmacêutico junto ao uso dos BDZ's, pode ser útil no momento da dispensação quando são ofertadas orientações precisas sobre a prescrição e tratamento, além do acompanhamento do usuário em seu tratamento farmacológico (OLIVEIRA; LOPES; CASTRO, 2015). Ainda nesse sentido, o trabalho do farmacêutico junto ao médico pode ser útil na "desprescrição" de BZD, o que constitui um desafio para a saúde pública, necessitando 
de disponibilidade dos profissionais em investir nessa prática, dos usuários em aceitá-la, além da necessária interlocução entre os envolvidos para a segurança da descontinuação do tratamento.

Contudo, é preocupante o uso irracional de BDZ's, pois os usuários desconhecem que o mesmo medicamento que pode lhes ajudar a aliviar o estresse e a insônia, dependendo da dose e tempo de uso, implica em alterações negativas à saúde. O conhecimento sobre o uso de medicamentos também relaciona-se com o tempo de uso dos BDZ's e a repercussão na vida dos usuários, pois quando o usuário tem conhecimento suficiente a respeito do medicamento ele pode repensar o seu uso e optar pelo uso a curto prazo. Nesse sentido, o farmacêutico e a equipe multiprofissional podem auxiliar o usuário a optar pelo uso quando esse for imprescindível, na tentativa de reduzir os riscos associados ao uso de BZD.

\section{CONSIDERAÇÕES FINAIS}

Os resultados encontrados reforçam a necessidade e a importância do acompanhamento dos usuários de psicofármacos nos serviços de saúde. Também permitem observar que o conhecimento desses sujeitos com relação aos medicamentos que utilizam, contribuem para a otimização da terapia, no que se refere à obtenção dos efeitos terapêuticos e uso dos medicamentos estritamente necessários, o que reflete de forma positiva na saúde do usuário, além de reduzir gastos com saúde.

O farmacêutico pode contribuir para o uso racional desses medicamentos, trabalhando individualmente com o usuário a partir da avaliação clínica dos medicamentos em uso, inclusive por meio de visitas domiciliares, sendo também necessária a interação com os prescritores para a manutenção do tratamento. Ainda, no matriciamento de equipes, o profissional pode identificar situações particulares de usuários, compartilhando conhecimento e experiências com os demais profissionais de saúde. Além disso, práticas alternativas e complementares ao uso de psicofármacos devem ser estimuladas, como modo de reduzir o uso irracional de medicamentos e promover saúde de modo mais natural.

Assim, se faz necessário o planejamento e implementação de estratégias em conjunto da equipe multiprofissional da APS, buscando interlocução entre o farmacêutico e demais profissionais, com vistas à resolutividade de casos, no contexto do SUS.

\section{REFERÊNCIAS}

ALVARENGA, J. M.; FILHO, A. I. L.; GIACOMINI, K.C. et al. Uso de benzodiazepínicos entre idosos: o alívio de "jogar água no fogo", não pensar e dormir. Revista Brasileira de Geriatria e Gerontologia, v. 18, n. 2, p. 249-258, 2015. Disponível em: https://bit.ly/2XDFxqg. Acesso em: 13 maio 2018. 
BRASIL. Ministério da saúde. Portaria 2.436 de 21 de setembro de 2017. Aprova a Política Nacional de Atenção Básica, estabelecendo a revisão de diretrizes para a organização da Atenção Básica, no âmbito do Sistema Único de Saúde (SUS). Diário Oficial da União. 22 set. 2017.

CARDOSO, L.; MIASSI, A.I.; GALERA, A. S. F. et al. Grau de adesão e conhecimento sobre tratamento psicofarmacológico entre pacientes egressos de internação psiquiátrica. Revista latino-americano de enfermagem, v. 19, n. 5, p. 9, 2011. Disponível em: https://bit.ly/3aeC7gr. Acesso em: 19 jun. 2018.

CARVAlHO, A. L. M.; LEOPOLDINO, R. W. D.; SILVA, J. E. G. S. et al. Adesão ao tratamento medicamentoso em usuários cadastrados no Programa Hiperdia no município de Teresina (PI). Ciências \& Saúde Coletiva, v.17, n.7, p. 1885-1892, 2012. Disponível em: https://bit.ly/2VbAoEg. Acesso em: 25 Out 2018.

CZARNOBAY, Y. J. Adesão ao uso de psicofármacos pelo portador de transtorno mental: percepções do enfermeiro (dissertação). Universidade Federal do Paraná, Curitiba, 2015. Disponível em: https://bit.ly/2VbRDoQ. Acesso em: 10 jun. 2018.

COUTINHO, F. H. P.; SOUZA, I. M. C. Percepção dos indivíduos com hipertensão arterial sobre sua doença e adesão ao tratamento medicamentoso na estratégia de saúde da família. Revista Baiana de Saúde Pública, v. 34, n. 2, p. 397-411, 2011. Disponível em: https://bit.ly/3abm6aL. Acesso em: 25 maio 2018.

FARIAS, A. I.; NETO, P. R.; GUIDONI, C. M. et al. Análise de medicamentos potencialmente inapropriados para idosos contidos na relação municipal de medicamentos essenciais (REMUME) de Divinópolis-MG. Journal of Applied Pharmaceutical Sciences, v. 2, n. 1, p. 48-69, 2015. Disponível em: https://go.aws/34Rgllf. Acesso em: 8 set. 2018.

FERREIRA, F. M.; CRUZ, M..J.; SANTOS, D. F. et al. Fatores relacionados à adesão farmacoterapêutica de pacientes hipertensos acolhidos na Estratégia Saúde da Família. Revista de Atenção Primária à Saúde, v. 16, n. 3, p. 258-268, 2013. Disponível em: https://bit.ly/3eqKPvg. Acesso em: 9 jun. 2018.

FIRMINO, K.F.; ABREU, M. H. N. G.; PERINI, E. et al. Utillização de benzodiazepínicos no serviço municipal de saúde da cidade de Coronel Fabriciano, Minas Gerais. Ciência \& Saúde Coletiva, v. 17, n. 1, p. 10, 2012. Disponível em: https://bit.ly/2VbzK9N. Acesso em: 12 jun. 2018. 
GOMES, R.; PIMENTEL, V.; LOUSADA, M. et al. O novo cenário de concorrência na indústria farmacêutica brasileira. Complexo industrial de saúde. BNDES Setorial, v. 39, p. 97-134, 2014. Disponível em: https://bit.ly/2K8IWFx. Acesso em: 14 out. 2018.

GOWDA, G. S.; DAS, S.; NANJEGOWDA, R. B. Psychotropic implant can be a new hope in psychiatry. Asian Journal of Psychiatry, v. 30, p. 214-217, 2017. Disponível em: https://bit.ly/2KdIhm4. Acesso em: 5 ago. 2018.

GRUBER, J.; MAZON, L. M. A prevalência na utilização de medicamentos psicotrópicos no município de mafra: um estudo retrospectivo. Saúde e Meio Ambiente, v. 3, n. 1, p. 44-50, 2014. Disponível em: https://bit.ly/2VBe4Th. Acesso em: 4 de set. de 2017. Acesso em: 6 Set 2018.

GUERRA, C. C. M.; FERREIRA, F.; DIAS, M. et al. Perfil epidemiológico e prevalência do uso de psicofármacos em uma unidade referência para saúde mental. Revista de enfermagem UFPE, v. 7, n. 6, p. 444-451, 2013. Disponível em: https://bit.ly/2yjKV7r. Acesso em: 10 maio 2018.

INSTITUTO BRASILEIRO DE GEOGRAFIA E ESTATÍSTICA. População de Santa Rosa, 2017. Disponível em: https://bit.ly/2RJ4qx0. Acesso em: 26 maio 2018.

LEITE, I. R. C. Atenção Farmacêutica: Adesão ao tratamento (monografia). Faculdade de Ciências Farmacêuticas de Araraquara “Júlio de Mesquita Filho", Araraquara, 2013. 99f. Disponível em: http:// hdl.handle.net/11449/119596. Acesso em: 7 ago. 2018.

MIASSO, A. I.; FILHO, P. C. P.; BORGES, T. L. et al. Quality of life in Brazil's Family Health Strategy: Common Mental Disordes. Use os psychotropic Drugs and Sociodemographic Factors. Archives of Psychiatric Nursing, v. 31, n. 1, p. 68-72, 2017. Disponível em: https://bit.ly/3ekpcwu. Acesso em: 8 jun. 2018.

MINAYO, M. C. S. Pesquisa social: teoria, método e criatividade. 29. ed. Petrópolis, RJ: Vozes, 2010.

NICOLINO, P. S.; VEDANA, K. G. G.; MIASSO, A. I. et al. Esquizofrenia: adesão ao tratamento e crenças sobre o transtorno e terapêutica medicamentosa. Revista Escola de Enfermagem, v. 3, n. 45 , p. 708-715, 2011. Disponível em: https://bit.ly/2VaIInI. Acesso em: 4 jul. 2018. 
OLIVEIRA, J. D. L.; LOPES, L. A. M.; CASTRO, G. F. P. Uso indiscriminado dos benzodiazepínicos: a contribuição do farmacêutico para um uso consciente. Revista transformar, 2015. Disponível em: https://bit.ly/2VEtyGu. Acesso em: 7 set. 2018.

OENNING, D.; OLIVEIRA, B. V.; BLATT, C. R. Conhecimento dos pacientes sobre os medicamentos prescritos após consulta médica e dispensação. Ciências \& Saúde Coletiva, v. 6, n. 7, 2011. Disponível em: https://bit.ly/3a9a81n. Acesso em: 6 maio 2018.

PREFEITURA MUNICIPAL DE SANTA ROSA. Lei 2.912 de 27 de dezembro de 1995. Cria a Fundação Municipal de Saúde de Santa Rosa - FUMSSAR - e dá outras providências. Disponível em https://bit.ly/2ygsNLA. Acesso em: 22 ago. 2018.

PRUDÊNCIO, F. A.; NOGUEIRA, L. T. Conhecimento de idosas sobre o uso de psicotrópicos. Revista da Rede de Enfermagem, v 14, n.1, p. 130-138, 2013. Disponível em: https://bit.ly/2VdYPRq. Acesso em: 22 ago. 2017.

SILVA, V. P.; BOTTI, N. C. L.; OLIVEIRA, V. C. et al. Perfil epidemiológico dos usuários de benzodiazepínicos na atenção primária à saúde. Revista de enfermagem do Centro Oeste mineiro, v. 5 , n. 1, p. 1393-1400, 2015. Disponível em: https://bit.ly/34GJpIm. Acesso em: 9 Jun 2018.

SOARES, J. C. R.; CAMARGO, J. R. A autonomia do paciente no processo terapêutico como valor para a saúde. Interface, v, 11, n. 21, p. 65-78, 2007. Disponível em: https://bit.ly/2VbSw0E. Acesso em: 4 de ago. de 2017.

SOLIS, C. X. B. Proposta de intervenção para reduzir o uso de benzodiazepínicos na equipe 04 da Unidade Básica de Saúde Vila Pinho em Belo Horizonte MG. Trabalho de conclusão de curso. Universidade Federal de Minas Gerais. 2016. Disponível em: https://bit.ly/3cs3LIh.

SOUTO, S. M. T. et al. Qualidade de vida de idosos usuários de benzodiazepínicos. Revista Atenção à Saúde, v. 15, n. 52, p. 96-101, 2017. Disponível em: https://bit.ly/2VeF3VK. Acesso em: 7 set. 2018.

SOUZA, C.; VEDANA, K. G. G.; MERCEDES, B. P. C. et al. Transtorno bipolar e medicamentos: adesão, conhecimento dos pacientes e monitorização sérica do carbonato de lítio. Revista Latino-Americana de Enfermagem, v. 21, n. 2, 2013. Disponível em: https://bit.ly/2ym2dk4. Acesso em: 7 ago. 2018 
